\title{
Relación entre el Burnout Académico y la Autoestima, en estudiantes de pregrado de Medicina $^{2}$
}

\author{
Vanessa Katherine Benavides Mora \\ Psicóloga \\ Universidad de Nariño \\ Correo electrónico: vanessakbenavidesm@gmail.com
}

Angie Johana Palacios Díaz

Psicóloga

Universidad de Nariño. Pasto, Colombia

Correo electrónico: angiepalaciosdiaz@gmail.com

\section{Christian Alexander Zambrano Guerrero}

Magister en Educación desde la Diversidad

Universidad de Nariño. Pasto, Colombia

Correo electrónico: cazambranopsi@gmail.com
Recibido: 23/10/2018

Evaluado: 06/12/2018

Aceptado: $15 / 12 / 2018$

\section{Resumen}

El objetivo del presente trabajo fue determinar la relación entre burnout académico y autoestima en estudiantes de pregrado de Medicina de San Juan de Pasto- Colombia. Estuvo orientado por el paradigma cuantitativo de tipo descriptivo correlacional, participaron 184 estudiantes de segundo, cuarto, sexto, octavo y décimo semestre, con edades entre los 17 a 40 años. Se empleó el Cuestionario Maslach Burnout Inventory-Student Survey (MBI-SS) y la Escala de autoestima de Rosenberg. En cuanto a resultados, se destaca que en las sub escalas de burnout académico no existieron diferencias estadísticamente significativas según el género; en la variable de autoestima no se encontraron diferencias por género ni semestre. Se pudo notar la existencia de una relación inversa entre autoestima y las sub escalas de agotamiento emocional y cinismo; mientras que la relación con eficacia académica fue directa, demostrando que estudiantes con niveles elevados de autoestima contarán con una menor posibilidad de presentar el síndrome.

Palabras clave

Burnout académico, autoestima, medicina, estudiantes universitarios.

2 Para citar este artículo: Benavides-Mora, V.K, Palacios-Díaz, A.J. \& Zambrano, C.A. (2020). Relación entre el Burnout Académico y la Autoestima, en estudiantes de pregrado de Medicina. Informes Psicológicos, 20(1), pp.19-31 http:// dx.doi.org/10.18566/infpsic.v20n1a02 


\title{
Relationship between academic burnout and self-esteem in undergraduate medical students
}

\begin{abstract}
The purpose of this work was to determine the relationship between academic burnout and self-esteem in undergraduate medical students in San Juan de Pasto, Colombia. This was a descriptive correlational study with the participation of 184 students in their second, fourth, sixth, eighth and tenth semesters, aged between 17 and 40 years. The Maslach Burnout Inventory-Student Survey (MBI-SS) and the Rosenberg Self-Esteem Scale were used. There were no statistically significant differences in the sub-scales of academic burnout according to gender. Similarly, no differences were found by gender or semester in the variable of self-esteem. It was possible to confirm the existence of an inverse relationship between self-esteem and the subscales of emotional exhaustion and cynicism, while the relationship with academic effectiveness was direct. This showed that students with high levels of self-esteem have a lower chance of presenting the syndrome.
\end{abstract}

Keywords

Academic burnout, self-esteem, medicine, university students.

\section{Relação entre o burnout acadêmico e a autoestima em estudantes de graduação em medicina}

\section{Resumo}

0 objetivo deste trabalho foi determinar a relação existente entre 0 burnout acadêmico e a autoestima em estudantes de graduação em medicina de San Juan de Pasto - Colômbia. A pesquisa esteve orientada pelo paradigma quantitativo de tipo correlacional descritivo, neste estudo tomaram parte 184 estudantes de segundo, quarto, sexto, oitavo e décimo semestre, com idades entre os 17 e os 40 anos. Foi implementado o questionário Maslach Burnout Inventory-Student Survey (MBI-SS) e a escala de autoestima de Rosenberg. Quanto aos resultados, destaca-se o fato de que nas subescalas de burnout acadêmico não houve diferencias estatisticamente relevantes relacionadas ao gênero; na variável de autoestima também não houve diferencias relacionadas ao gênero ou ao semestre. Observou-se a existência de uma relação inversa entre a autoestima e as subescalas de exaustão emocional e cinismo; enquanto a relação com a efetividade acadêmica foi direta, demonstrando que os alunos com altos níveis de autoestima terão menor chance de apresentar a síndrome.

Palavras chave

Burnout acadêmico, autoestima, medicina, estudantes universitários. 


\section{ntroducción}

Para Gil-Monte (2003) el burnout se refiere al avanzado desgaste profesional o cansancio emocional. Dentro de los estudios más destacados se encuentran los realizados por Maslach y Jackson (1981) quienes lo entienden como un síndrome causado por las excesivas demandas del trabajo. Señalan que cuenta con tres dimensiones que son: agotamiento emocional, siendo las sensaciones de sobreesfuerzo físico y emocional; despersonalización, como las actitudes generadas por una carga laboral estresante, adoptando respuestas cínicas hacia las personas que le rodean; reducida realización personal, como la pérdida de confianza en la realización personal y bajos niveles de auto concepto. Los síntomas del síndrome se presentan principalmente en aquellos profesionales que trabajan con seres humanos (Vizcarra, Llaja, \& Talavera, 2015). Para realizar un estudio pertinente sobre el burnout, se hace necesario considerar que en la actualidad, las demandas del trabajo son cada vez mayores, presentándose este síndrome de manera particular entre las profesiones o de acuerdo a los roles que se puede desempeñar en una organización, debido a las jornadas laborales con poca remuneración, sin pago de horas extra y con escasa o nula motivación hacia el trabajo o reconocimiento por el logro de los objetivos del profesional (Vega, Gómez, Caballero \& Contreras, 2017; Vieco \& Abello, 2014).

Tradicionalmente, se ha estudiado el síndrome de burnout en profesionales de la medicina o la enfermería. Sin embargo, se ha demostrado que también se presenta de manera estable en otras profesiones. Actualmente, es de interés el estudio del burnout en estudiantes, debido al agotamiento por las exigencias del medio, presentando actitudes distantes e ineficacia hacia las actividades académicas (Martínez, Marques \& López da Silva, 2002).

Por lo tanto, cuando los estudiantes ingresan a un periodo de práctica profesional en una organización donde desempeñan un rol específico, desarrollan actividades que requieren tiempo, esfuerzo y cumplen objetivos, encontrándose en constante evaluación y siendo incentivados por los profesores, quienes supervisan la labor, experimentan elevados niveles de estrés y ansiedad, lo cual ocasiona en muchos de los casos bajo desempeño académico, agotamiento físico y emocional, así como también una pérdida en los niveles de autoestima (Caballero, Breso \& González, 2015). Por lo tanto, el burnout académico en estudiantes universitarios se concibe como una forma de estrés que puede afectar el desarrollo, compromiso y satisfacción con la formación académica y salud psicosocial.

El síndrome está mediado por las condiciones relacionadas con las actividades del estudiante, las características psicosociales de la universidad y aspectos personales como los recursos psicológicos con los que cuenta, tales como estrategias de afrontamiento y resolución de problemas (Caballero, Palacio \& Hederich, 2012). El burnout académico, se manifiesta en tres dimensiones que son: agotamiento (no poder dar más de sí mismo), cinismo (actitud negativa y desinterés por el estudio) y autoeficacia académica (percepción sobre la competencia en los estudios) (Schaufeli, Martínez, Marques, Salanova \& Bakker, 2002). 
Caballero, Abello y Palacio (2007) destacan que el $41.6 \%$ de los estudiantes de una universidad privada de Barranquilla, Colombia, presentan burnout académico. Por otra parte, Martínez y Salanova (2003) demostraron la diferencia según el género en la universidad Jaume I de Castellón, España, señalando que las mujeres presentan menos actitud negativa o cinismo frente al estudio y a su vez se perciben más eficaces que los hombres. Contrario a lo anterior, Rosales (2012) encontró que la mayoría de estudiantes de medicina de la Universidad de Ciencias Médicas de Holguín, Cuba, presentan niveles del síndrome y las mujeres, mayor incidencia de burnout académico. Estrada, De la Cruz, Bahamón, Pérez y Cáceres (2018) evidenciaron que la prevalencia del síndrome es mayor en los primeros y últimos semestres en estudiantes de Barranquilla.

En este sentido, se ha prestado interés a las variables psicológicas, responsables de los cambios personales en el burnout, donde destacan la autoestima, definida como la autovaloración y consideración del sí mismo (González, Souto, Freire, Fernández \& González, 2016; Musitu \& Vera, 2000, citados por Larrota, Sánchez \& Sánchez, 2016). Según Cabanach, Souto, Fernández y Freire (2011), la baja autoestima genera auto rechazo, insatisfacción y desajuste personal, por lo cual, la potenciación de este atributo actuaría como un factor protector ante el estrés, propiciando el óptimo afrontamiento.

González et al. (2016) realizaron un estudio con estudiantes de fisioterapia en España, donde se evidenció que entre la autoestima y el burnout académico existe una relación estadísticamente significativa, destacando que a mayor nivel de autoestima el estudiante presentará menores niveles en las dimensiones agotamiento emocional y cinismo, mientras que la correlación entre autoestima y eficacia académica es positiva. En concordancia, Del Ángel, Santes, Aldrete, Lavoignet y Meléndez (2009), realizaron un estudio en una universidad pública del Estado de Veracruz, México, a partir del cual se encontró que la tendencia a presentar burnout se encuentra latente en aquellas personas con baja autoestima.

Estudiar el burnout académico es relevante debido a que los estudiantes de la salud manifiestan elevados niveles de estrés por el alto y creciente nivel de exigencia de la carrera y por la complejidad en las jornadas de clase y prácticas. De igual forma, se enfrentan a la presión social del trabajo directo con seres humanos, debido al impacto positivo o negativo de las acciones que realicen (Ortega, Ortiz \& Martínez, 2014). Lo anterior puede generar agotamiento físico, mental y emocional, facilitando el desarrollo de actitudes negativas hacia el estudio y perjudicando la percepción de las competencias académicas, afectando la satisfacción y rendimiento académico (Caballero et al., 2007).

Teniendo en cuenta lo anterior, el objetivo del estudio es determinar la relación entre burnout académico y autoestima en estudiantes de pregrado de la Facultad de Ciencias de la Salud de una universidad pública de San Juan de Pasto-Colombia, dado que a partir de dicho análisis se hará posible formular estrategias de evaluación e intervención desde la psicología identificando maneras para reducir o prevenir el burnout académico y fortalecer el bienestar psicosocial, así como potenciar el desempeño académico en las labores estudiantiles que día a día se realizan tanto en el contexto universitario como en 
los diferentes contextos de salud donde se desarrollan prácticas académicas y profesionales.

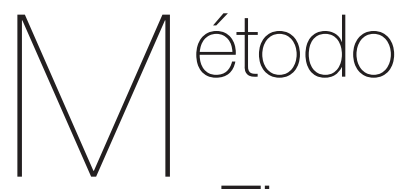

\section{Tipo de estudio}

El presente estudio se realizó bajo un paradigma cuantitativo de tipo correlacional con corte transversal, es decir, recolectando la información en un solo momento en el tiempo, a partir de lo cual se correlacionaron las variables de estudio (Hernández-Sampieri, Fernández \& Baptista, 2010).

\section{Instrumentos}

Se utilizó el Cuestionario Maslach Burnout Inventory-Student Survey (MBI-SS) validado en el contexto académico colombiano por Hederich-Martínez y Caballero-Domínguez (2016), compuesto por tres sub-escalas que son: agotamiento, cinismo y autoeficacia académica. Consta de 14 ítems que se responden con una escala Likert de 7 puntos que va de 0 (nunca) a 6 (siempre). Cada sub-escala presenta una buena consistencia interna: agotamiento $(\mathrm{a}=.77)$, cinismo $(\mathrm{a}=.72) \mathrm{y}$ autoeficacia académica $(a=.82)$.

Se aplicó la Escala de autoestima de Rosenberg desarrollada por Rosenberg (1965) y validada en Colombia por Gómez et al. (2016), encargada de evaluar la autoestima en adolescentes. Consta de diez ítems centrados en los sentimientos de respeto y aceptación de sí mismo/a y se responden en una escala Likert de cuatro puntos que va de 1 (muy de acuerdo) a
4 (totalmente en desacuerdo). La puntuación total, oscila entre 10 y 40. Presenta una consistencia interna $(\mathrm{a}=.87)$ y una confiabilidad test- retest de .72 .

\section{Participantes}

Participaron estudiantes de la Facultad de Ciencias de la Salud de una universidad pública ubicada en San Juan de Pasto. La muestra se determinó mediante un muestreo por conglomerados bietápico. En la primera etapa se seleccionaron los semestres y en la segunda etapa los estudiantes de cada semestre mediante muestreo aleatorio simple con un nivel de confianza de $95 \%$ y un error del 5\%. La muestra final estuvo conformada por 184 estudiantes (98 mujeres y 86 hombres) de segundo, cuarto, sexto, octavo y décimo semestre con edades entre los 17 y 40 años.

\section{Procedimiento}

Una vez se obtuvieron los respectivos permisos institucionales para realizar el estudio, los participantes diligenciaron el consentimiento informado, destacando la participación voluntaria, confidencial y sin riesgos ni beneficios directos. Posteriormente, se realizó la aplicación de los instrumentos de medición. En tercer lugar, se sistematizaron los datos en Microsoft Excel y se analizó la información mediante el paquete estadístico IBM SPSS Statics 23, que permitió obtener puntuaciones, agrupaciones y porcentajes de los resultados. Inicialmente, se interpretaron los datos de la variable burnout académico, seguida de la variable autoestima y finalmente se realizó una correlación entre ambas. 


\section{Consideraciones éticas}

El estudio se desarrolló bajo la normatividad de la Ley 1090 de 2006, donde se establece el Código Deontológico y Bioético del psicólogo, y se estipulan los principios éticos de Justicia, Libertad, Beneficencia y No Maleficencia para salvaguardar los derechos y bienestar de los colaboradores. También, se tuvo en cuenta la Resolución 8430 de octubre 4 de 1993, para considerar la investigación con riesgo mínimo al no manipular la conducta de los participantes. Es importante mencionar que se solicitó la autorización y colaboración de los directivos del Departamento de Psicología y de la Facultad de Ciencias de la Salud. El trabajo de campo fue realizado y supervisado por profesionales con conocimiento y experiencia para salvaguardar la integridad de los participantes y garantizar su bienestar.

Pesultados

\section{Burnout Académico}

Para el análisis de datos de la variable Burnout Académico se calcularon los estadísticos descriptivos de cada sub escala (ver Tabla 1), a partir de los cuales se afirma que los estudiantes presentan en promedio un nivel alto de Agotamiento Emocional, nivel medio- alto de cinismo y un nivel medio- bajo de Eficacia Académica.
Tabla 1

Estadísticos Descriptivos para las sub escalas del Cuestionario MBI ES.

\begin{tabular}{lcccccc}
\hline \multicolumn{7}{c}{ Estadísticos } \\
\hline Sub escala & Media & $\begin{array}{c}\text { Desv. } \\
\text { Estándar }\end{array}$ & Mediana & Moda & Mín. & Máx. \\
\hline $\begin{array}{l}\text { Agotamiento } \\
\text { Emocional }\end{array}$ & 3.31 & 1.2 & 3.2 & 3 & 0 & 6.0 \\
$\begin{array}{l}\text { Cinismo } \\
\text { Eficacia } \\
\text { Académica }\end{array}$ & 1.42 & 1.2 & 1.2 & 0 & 0 & 5.3 \\
\hline
\end{tabular}

Mediante la prueba Kolmogrov Smirnov se comprobó que las puntuaciones de Agotamiento Emocional se distribuyen de forma normal con un $p$ valor $=.062$ y las puntuaciones de Cinismo y Eficacia Académica no se distribuyen de forma normal con un $p$ valor $=.00$.

\section{Análisis de datos por género}

Teniendo en cuenta los datos obtenidos con la prueba de normalidad, para determinar si existen diferencias entre hombres y mujeres, se utilizó la prueba T de Student para la sub escala Agotamiento Emocional y la prueba $U$ de Mann Whitney para las sub escalas Cinismo y Eficacia Académica.

\section{Agotamiento Emocional}

Mediante la comparación de medias entre hombres y mujeres se evidencia que el puntaje promedio de los hombres (3.36) y el de las mujeres (3.24) no varía en gran medida y los dos presentan un nivel alto de Agotamiento Emocional. 
La prueba de Levene permitió afirmar que las varianzas entre hombres y mujeres en la sub escala de Agotamiento Emocional son iguales. Por su parte, la significancia de la prueba $T$ de student indica que no hay diferencias estadísticamente significativas según el género con un $p$ valor $=.482$.

\section{Cinismo y Eficacia Académica}

Según la media de las dos sub escalas, tanto hombres como mujeres presentan un nivel medio alto en la sub escala Cinismo con un puntaje promedio de 1.47 y 1.37 respectivamente. De igual manera hombres y mujeres presentan un nivel medio- bajo en la sub escala Eficacia Académica con promedios de 4.10 y 4.00 respectivamente.

A partir de los datos arrojados por la prueba $U$ de Mann-Whitney se afirma que no existen diferencias estadísticamente significativas entre hombres y mujeres para las sub escalas de Cinismo ( $p$ valor $=.81) \mathrm{y}$ Eficacia Académica (.64).

\section{Análisis de datos por semestre}

Para determinar si existen diferencias en la puntuación de las tres sub escalas de acuerdo al semestre de los participantes, se utilizaron las pruebas ANOVA para la sub escala de Agotamiento Emocional y la prueba Kruskal-Wallis para las sub escalas Cinismo y Eficacia Académica.

\section{Agotamiento Emocional}

Al comparar las medias de cada semestre (ver Tabla 2) se evidencia que todos los participantes presentan un nivel alto de Agotamiento Emocional con un leve incremento en las puntuaciones de octavo y décimo semestre.

Tabla 2

Promedio de la sub escala Agotamiento Emocional según cada semestre

\begin{tabular}{cccc}
\hline Semestre & N & Media & Desv. Estándar \\
\hline 2 & 45 & 3.23 & 1.10 \\
4 & 38 & 3.23 & 1.16 \\
6 & 71 & 3.21 & 1.28 \\
8 & 23 & 3.81 & 1.32 \\
10 & 7 & 3.48 & .30 \\
\hline
\end{tabular}

Según la prueba Anova se afirma que en esta sub escala no existen diferencias estadísticamente significativas de acuerdo al semestre, con un $p$ valor $=.28$.

\section{Cinismo y Eficacia Académica}

De acuerdo a las medias (ver Tabla 3), en la sub escala Cinismo, los participantes de segundo y cuarto semestre presentan un nivel medio-bajo, los participantes de sexto y décimo semestre presentan un nivel medio- alto y los participantes de 8 semestre presentan un nivel alto.

Por su parte, en cuanto a la sub escala de Eficacia Académica se evidencia que los participantes de todos los semestres presentan un nivel medio-bajo. 
pp 19-31 Vanessa Katherine Benavides Mora, Angie Johana Palacios Díaz y Christian Alexander Zambrano Guerrero

Tabla 3

Promedio de la sub escalas Cinismo y Eficacia Académica en hombres y mujeres por semestre

\begin{tabular}{ccccc}
\hline & \multicolumn{2}{c}{ Cinismo } & \multicolumn{2}{c}{ Efic. Académica } \\
\hline Semestre & Media & $\begin{array}{c}\text { Desv. } \\
\text { Estándar }\end{array}$ & Media & $\begin{array}{c}\text { Desv. } \\
\text { Estándar }\end{array}$ \\
\hline 2 & 1.0 & 1.0 & 3.9 & .7 \\
4 & 1.0 & .9 & 4.2 & .8 \\
6 & 1.4 & 1.2 & 4.1 & .9 \\
8 & 2.5 & 1.1 & 3.9 & .7 \\
10 & 1.6 & 1.5 & 4.5 & 1.0 \\
\hline
\end{tabular}

Los datos arrojados por la prueba Kruskal-Wallis y el nivel de significancia permitieron determinar que las diferencias son estadísticamente significativas según el semestre solamente en la sub escala de Cinismo con un $p$ valor $=.00$ mientras que la sub escala de Eficacia Académica con un $p$ valor $=.15$ indica que no existen diferencias.

Teniendo en cuenta que una de las sub escalas presenta diferencias según el semestre, el análisis de la variable Burnout se realizará por semestres.

\section{Niveles de Burnout por semestre}

Teniendo en cuenta que una persona presenta el síndrome de burnout académico cuando sus niveles son altos o muy altos en las sub escalas de Cinismo y Agotamiento Emocional y bajos o muy bajos en la escala de eficiencia académica, los resultados obtenidos por semestre se presentan en la Tabla 4.
Tabla 4

Estudiantes que presentan síndrome de burnout académico.

\begin{tabular}{cccc}
\hline Semestre & $\begin{array}{c}\text { Estudiantes } \\
\text { evaluados }\end{array}$ & $\begin{array}{c}\text { Estudiantes que } \\
\text { presentan Burnout }\end{array}$ & $\%$ \\
\hline 2 & 45 & 4 & $8.9 \%$ \\
4 & 38 & 8 & $21.1 \%$ \\
6 & 71 & 5 & $7 \%$ \\
8 & 23 & 3 & $13 \%$ \\
10 & 7 & 1 & $14.3 \%$ \\
\hline
\end{tabular}

\section{Autoestima}

Para el análisis de datos de la variable Autoestima se calcularon los estadísticos descriptivos de la escala total evidenciando que los estudiantes presentan en promedio un nivel elevado de autoestima con un puntaje promedio de 33.17.

\section{Comparación de grupos}

Para realizar el análisis de datos por género y por semestre se aplicó la prueba Kolmogrov Smirnov, mediante la cual se pudo comprobar que los datos no se distribuyen de forma normal, con un $p$ valor $=.00$. Por lo tanto, para comparar los datos por género se utilizó la prueba U de Mann-Whitney y para comparar los datos por semestre se utilizó la prueba Kruskal-Wallis.

\section{Análisis de datos por sexo}

Según la media de cada género, se afirma que en promedio los dos presentan un nivel elevado (normal) de autoestima con puntajes promedios de 33.02 para los hombres y 33.31 para las mujeres.

A partir de los datos arrojados por la prueba $U$ de Mann-Whitney se afirma que 
no existen diferencias estadísticamente significativas de acuerdo al género de los participantes con un $p$ valor $=.94$.

\section{Análisis de datos por semestre}

De acuerdo a los datos se puede afirmar que los participantes de todos los semestres, en promedio presentan un nivel elevado de autoestima (ver Tabla 5).

Tabla 5

Promedio de Autoestima por semestre

\begin{tabular}{ccc}
\hline Semestre & Media & Desv. Estándar \\
\hline 2 & 33.49 & 3.89 \\
4 & 33.63 & 5.62 \\
6 & 32.68 & 5.37 \\
8 & 33.00 & 3.45 \\
11 & 34.29 & 6.47 \\
\hline
\end{tabular}

Mediante la prueba Kruskal-Wallis se afirma que no existen diferencias estadísticamente significativas en la variable autoestima de acuerdo al semestre que cursan los participantes con un $p$ valor $=.61$.

Teniendo en cuenta que no existen diferencias por semestre ni por género, el análisis de los niveles de autoestima se hará de forma general para todos los participantes.

\section{Niveles de autoestima}

A partir del análisis de frecuencias se evidenció que la mayoría de estudiantes (79\%) tiene un nivel elevado de autoestima, el cual es considerado como normal. Por otra parte, se evidencia que el $11.4 \%$ de los participantes presentan un nivel medio de autoestima, el cual no es grave, pero si requiere trabajarse. Finalmente, sólo el $8.7 \%$ de estudiantes presentan niveles bajos de esta variable.

Relación entre las sub escalas del Cuestionario Maslach Burnout Inventory-Student Survey (MBI ES) y Autoestima

Para analizar la correlación entre las sub escalas de Agotamiento Emocional, Cinismo y Eficacia Académica con la variable Autoestima, se utilizó el índice de correlación de Spearman, a partir del cual se logró evidenciar que la variable autoestima y las sub escalas de burnout si bien presentan índices de correlación bajos, son estadísticamente significativas. El Agotamiento Emocional correlaciona negativamente $(r=-.26$ y $p$ valor $=.00)$ así como la sub escala Cinismo $(r=-.39$ y $p$ valor $=.00$ ), lo cual quiere decir que las personas con niveles más altos de autoestima presentan niveles más bajos en estas dos sub escalas. Por otra parte, la eficacia académica correlaciona de forma positiva con la autoestima ( $r=.47$ y $p$ valor $=.00$ ), de tal forma que una persona con alta autoestima presentará niveles altos de la sub escala.

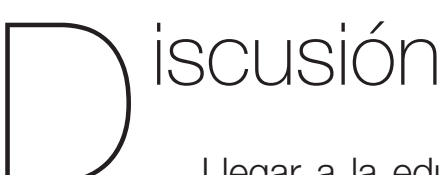

Llegar a la educación superior resulta un logro importante en la vida personal y la culminación de ésta se ha convertido en una etapa fundamental en la vida profesional, en este proceso, el individuo adquiere los conocimientos y habilidades necesarias para ejercer la profesión a desarrollar en 
el futuro. Sin embargo, el contexto y las exigencias de las instituciones son constantemente descritos como generadores de altos niveles de estrés (Estrada et al., 2018). Por lo tanto, el estudiante universitario se expone diariamente a desafíos y exigencias dadas por las condiciones propias del contexto educativo, en donde los procesos de aprendizaje y rendimiento académico se convierten en fuentes de estrés (Caballero et al., 2007).

Cuando la persona no logra adaptarse a estos eventos y no cuenta con estrategias adecuadas de afrontamiento, podría encontrarse física, mental y emocionalmente exhausto y con una sensación de no poder dar más de sí mismo en la academia (agotamiento emocional), una actitud negativa académica evidenciada a través de la autocrítica, desvalorización, pérdida del interés, de la trascendencia y valor frente al estudio (cinismo) y la percepción del estudiante sobre la competencia en el estudio (eficacia académica) (Gil-Monte, 2003; Hederich-Martínez \& Caballero-Domínguez, 2016).

Siendo el burnout una variable psicológica de interés, el objetivo del presente estudio fue evaluar el síndrome de burnout académico y la autoestima en estudiantes de pregrado, con el fin de conocer la relación existente entre estas variables, para lo cual se utilizaron cuestionarios válidos y confiables en el contexto. Entre los hallazgos del estudio se destaca que no existieron diferencias estadísticamente significativas según el género en las sub escalas del cuestionario de burnout, que difiere de lo encontrado por Martínez y Salanova (2003) al manifestar que la prevalencia del burnout es mayor en hombres, especialmente en las subescalas de cinismo y eficacia académica lo que, en corto y mediano plazo, puede ocasionar el abandono de las actividades académicas lo cual, psicológicamente, puede implicar una sensación de abandono e incapacidad para cumplir objetivos así como la deserción de los estudios universitarios (Rosales, 2012).

Por su parte, autores como Cabanach, Fariña, Freire, González y Del Mar (2013) mencionan que existen diferencias de género en cuanto a la prevalencia de estos síntomas, las cuales están mediadas por las estrategias de afrontamiento a las que se recurre, confirmando que los hombres, ante situaciones que generan altos niveles de estrés como la sobre exigencia académica, a diferencia de las mujeres, suelen inclinarse por adoptar un pensamiento positivo o bien planificar y gestionar los recursos precisos para resolverlo. Mientras que, las mujeres parecen recurrir a estrategias como la búsqueda de apoyo social como estrategia para enfrentar el estrés percibido. En este sentido, es importante considerar dichos recursos para implementar estrategias de intervención que permitan al estudiante afrontar las exigencias propias del contexto universitario. Teniendo en cuenta el semestre en curso, solamente se encontraron diferencias en la subescala de cinismo, por lo cual, el análisis de la variable total se realizó por semestre.

Una persona presenta burnout cuando los niveles de agotamiento emocional y cinismo son altos y el nivel de eficacia académica es bajo; se encontró que la prevalencia es mayor en los estudiantes de cuarto semestre. Estos hallazgos guardan relación con lo encontrado por Estrada et al. (2018) quienes mencionan 
que el síndrome se presenta en un nivel moderado en estudiantes de primeros y últimos semestres, ya que, por lo general, los estudiantes universitarios inician esta nueva etapa con incertidumbres y pequeños niveles de estrés que incrementan progresivamente, puesto que la exigencia es mayor y la actividad académica se intensifica (Barraza, 2009). Sin embargo, en el presente estudio, se encontró que, en los últimos semestres, la prevalencia de dicho síndrome es menor. Esto puede presentarse debido a que la muestra de estudiantes de $10^{\circ}$ semestre fue reducida en comparación con los otros semestres.

En la variable de autoestima no se encontraron diferencias por género ni por semestre contrario a lo expuesto por González, Valdez y Serrano (2003), quienes mencionan que la variable puede diferenciarse según el género, siendo las mujeres quienes presentan menores niveles. Respecto a los niveles de autoestima se encontró que la mayoría de estudiantes (79.9\%) presentan un nivel elevado. Este resultado es coherente con lo encontrado en el estudio de Chilca (2017), donde más de la mitad (52.3\%) de estudiantes de la Facultad de Ingeniería de la Universidad Tecnológica del Perú presentan un nivel alto, seguido del $41.9 \%$ que tienen un nivel de autoestima Medio Alto, y el 5.8\% de los estudiantes tienen un nivel de autoestima de "Medio bajo". Siendo la autoestima una de las principales variables, se resalta la existencia de otras que pueden aportar negativamente en el desarrollo de la vida universitaria; en primer lugar, el factor social, seguido del económico, los culturales y por último el nivel psicológico con que cada estudiante afronta la universidad (Caballero et al., 2007).
Finalmente, mediante el presente estudio se evidenció la existencia de una relación inversa entre autoestima y las sub escalas de agotamiento emocional y cinismo, y una relación directa con eficacia académica, de tal forma que estudiantes con niveles elevados de autoestima tendrán menor posibilidad de presentar burnout. En coherencia, González et al. (2016) señalan que entre la autoestima y el burnout académico existe una relación, destacando que, a mayor nivel de autoestima, el estudiante presentará menores niveles en las dimensiones agotamiento emocional y cinismo, mientras que la correlación entre autoestima y eficacia académica es positiva. Estos resultados permiten confirmar que la variable autoestima actúa posiblemente como un factor protector frente al síndrome de burnout (Aranda, Pando, Torres, Salazar \& Mares, 2015), lo cual invita a las instituciones universitarias a trabajar en el fortalecimiento de ésta para promover la salud mental de los jóvenes universitarios y prevenir la prevalencia de consecuencias psicológicas que afectan tanto al estudiante como a las diferentes organizaciones en las cuales se está inmerso.

La presencia del síndrome de burnout en estudiantes universitarios está mediada por variables como el apoyo social y emocional y las estrategias de afrontamiento, por esta razón es importante que las instituciones de educación superior presten atención a las variables personales y psicológicas necesarias para el óptimo desempeño y transversales al rendimiento académico, teniendo en cuenta que promoverla disminuye el riesgo al que se enfrentan los estudiantes de presentar estrés, síndrome de burnout y a la vez trastornos de mayor gravedad como ansiedad, depresión, entre otros. Además 
de reducir la posibilidad de la deserción académica. Finalmente, se reconoce que la ejecución de actividades de promoción orientadas al fortalecimiento de variables personales en la etapa universitaria permite la adquisición de herramientas y estrategias de afrontamiento que, en un futuro, se verán reflejadas positivamente el entorno laboral.

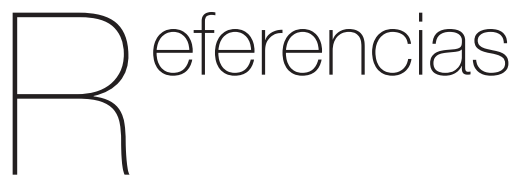

Aranda, C., Pando M., Torres, T., Salazar, J. \& Mares, F. (2015). Factores sociodemográficos y laborales, apoyo social, autoestima y Síndrome de Burnout, en trabajadores de tiendas de abarrotes de Guadalajara, México. Salud UDES, 2(1), 18-24.

Barraza, A. (2009). Estrés académico y burnout estudiantil. Análisis de su relación en alumnos de licenciatura. Psicogente, 12(22), 272-283.

Caballero, C., Abello, R. \& Palacio, J. (2007). Relación del burnout y el rendimiento académico con la satisfacción frente a los estudios en estudiantes universitarios. Avances en Psicología Latinoamericana, 25(2), 98-111.

Caballero, C., Breso, É. \& González, O. (2015). Burnout en estudiantes universitarios. Psicología desde el Caribe, 32(3), 24-44.

Caballero, C., Palacio, J. \& Hederich, C. (2012). El burnout académico: Prevalencia $y$ factores asociados en estudiantes universitarios del área de la salud de la ciudad de Barranquilla (Tesis doctoral). Universidad del Norte, Barranquilla.
Cabanach, R. G., Fariña, F., Freire, C., González, P., \& Del Mar, M. (2015). Diferencias en el afrontamiento del estrés en estudiantes universitarios hombres y mujeres. European Journal of Education and Psychology, 6(1), 19-32.

Cabanach, R., Souto, A., Fernández, R. \& Freire, C. (2011). Regulación emocional y burnout académico en estudiantes universitarios de Fisioterapia. Revista de Investigación en Educación, 9(2), 7-18.

Chilca, M. (2017). Autoestima, hábitos de estudio y rendimiento académico en estudiantes universitarios. Propósitos $y$ Representaciones, 5(1), 71-127.

Del Ángel, E. M., Santes, M. C., Aldrete, M., Lavoignet, B. \& Meléndez, S. (2009). Análisis sobre Burnout y Autoestima en Académicos de una Universidad Pública. Desarrollo Científico de Enfermería, 17(10), 435-439.

Estrada, H., De la Cruz, A., Bahamón, J., Pérez, J., \& Cáceres, M. (2018). Burnout académico y su relación con el bienestar psicológico en estudiantes universitarios. Revista Espacios, (39), 7-23.

Gil-Monte, P. (2003). El síndrome de quemarse por el trabajo (síndrome de burnout) en profesionales de enfermería. Revista Eletrônica InterAção Psy, 1(1), 19-33.

Gómez, M., Espada, J., Morales, A., Marchal, L., Soler, F. \& Vallejo, P. (2016). Adaptation, validation, reliability and factorial equivalence of the Rosenberg self-esteem scale in Colombian and Spanish population. The Spanish Journal of Psychology, 19, 1-12.

González, R., Souto, A., Freire, C., Fernández, R. \& González, L. (2016). La autoestima 
como variable protectora del burnout en estudiantes de fisioterapia. Estudios sobre Educación, (30), 95-113.

González, N., Valdez, J. \& Serrano, J. (2003). Autoestima en jóvenes universitarios. CIENCIA ergo-sum, Revista Cientifica Multidisciplinaria de Prospectiva, 10(2), 173-179.

Hernández, R, Fernández, C. \& Baptista, P. (2010). Metodología de la investigación. México: Quinta Ed. McGraw Hill Interamericana.

Hederich-Martínez, C. \& Caballero-Domínguez, C. (2016). Validación del cuestionario Maslach Burnout Inventory-Student Survey (MBI-SS) en contexto académico colombiano. Revista CES Psicología, 9(1), 1-15.

Larrota, R., Sánchez, L. \& Sánchez, J. (2016). Niveles de autoestima y uso de estrategias de afrontamiento en personas privadas de la libertad en un centro de reclusión de la ciudad de Bucaramanga. Informes Psicológicos, 16(1) 51- 64.

Martínez, I., Marques, A., Salanova, M. \& López, A. (2002). Burnout en estudiantes universitarios de España y Portugal. Un estudio croscultural. Ansiedad y Estrés, (8), 13-23.

Martínez, I. \& Salanova, M. (2003). Niveles de burnout y engagement en estudiantes universitarios. Relación con el desempeño y desarrollo profesional. Revista de Educación, (330), 361-384.

Maslach, C. \& Jackson, S. E. (1981). The measurement of experienced burnout. Journal of Organizational Behavior, 2(2), 99-113.
Ortega, M., Ortiz, G. \& Martínez, A. (2014). Burnout en estudiantes de pregrado de medicina y su relación con variables de personalidad. Terapia Psicológica, 32(3), 235-242.

Rosales, Y. (2012). Estudio unidimensional del síndrome de burnout en estudiantes de medicina de Holguín. Revista de la Asociación Española de Neuropsiquiatría, 32(116), 795-803.

Rosenberg, M. (1965). Rosenberg selfesteem scale (RSE). Acceptance and Commitment Therapy. Measures Package, 61. Woolongong, Australia: University of Wollongong.

Schaufeli, W., Martínez, I., Marques Pinto, A., Salanova, M., \& Bakker, A. (2002). Burnout and engagement in university students: A cross-national study. Journal of CrossCultural Psychology, 33(5), 464-481.

Vega, E., Gómez, J., Caballero, I. \& Contreras, F. (2017). Síndrome de burnout y calidad de vida laboral en personal asistencial de una institución de salud en Bogotá. Informes Psicológicos, 17(1), 87-105.

Vieco, G. \& Abello, R. (2014). Factores psicosociales de origen laboral, estrés y morbilidad en el mundo. Psicología desde el Caribe, 31(2), 354-385.

Vizcarra, M., Llaja, V. \& Talavera, J. (2015). Clima laboral, Burnout y perfil de personalidad: un estudio en personal asistencial de un hospital público en Lima. Informes Psicológicos, 15(2), 111- 126. 